\title{
EDITORIAL
}

Check for updates

\section{Reflecting on 20 years of progress}

\author{
This issue marks the 20th anniversary of Nature Reviews Cancer. On this milestone birthday \\ we both look back on the past 20 years of cancer research and look forward to the future.
}
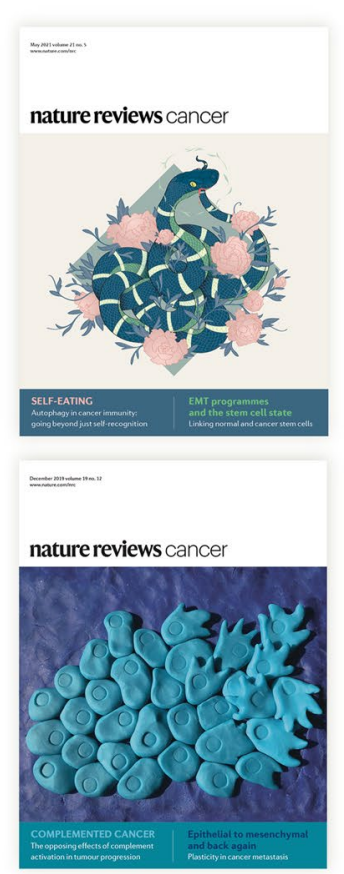

nature

REVIEWS

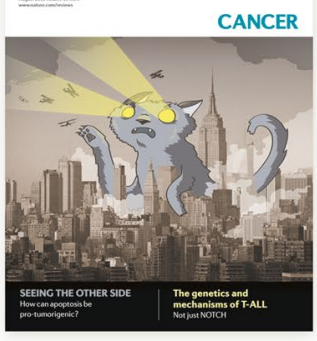

nature

REVIEWS

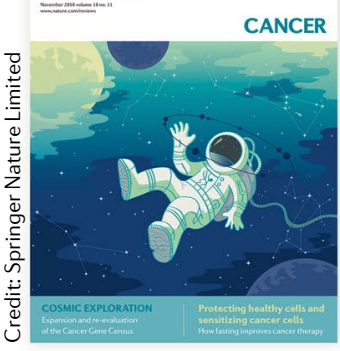

For those of us working and thinking about cancer research every day, it can sometimes seem like progress is slow. Although we all hoped that advances in preventing, detecting and treating cancer would come faster, much has been achieved in the past 20 years; some of these achievements, as well as future challenges, are the focus of articles in our 20th Anniversary issue and associated Collection.

It is somewhat remarkable to think that when this journal published its first issue in October 2001, the first tyrosine kinase inhibitor for cancer therapy, imatinib, had just been approved. Its development (along with that of trastuzumab 3 years before) heralded a new era in cancer therapy; $>100$ targeted therapies are now approved to treat many different cancer types. We now appreciate that targeted therapies are not the 'magic bullets's we once hoped they would be, and that resistance to targeted therapies is incredibly complex ${ }^{2}$. But, there have been steps towards making targeted therapies better, for example by using targeted protein degradation.

Not long after the journal started, The Cancer Genome Atlas (TCGA) and other cancer genome sequencing initiatives were launched. Despite the insights these efforts have provided, we still have much to learn about cancer genomes. The volume of data collected has allowed us to identify patterns (mutational signatures) and to study not just single mutations but the genomic context of each patient's tumour. Continuing on this theme, a systematic approach to understanding the noncoding genome regulatory landscape of individual tumours may also help us understand contexts.

In 2001, the importance of the tumour microenvironment (TME) was clear to many ${ }^{3}$, but in the past 20 years we have gained tremendous insights into how different cellular and structural components of the TME influence cancer biology. We also cannot ignore the surge of research into cancer immunology and the development of immunotherapies. Although not specifically covered in articles in this issue, these topics are addressed by many articles in the associated Collection.

Although the presence of altered metabolic pathways in tumours has been known for much longer than 20 years, the field experienced a resurgence of interest in the past $\sim 10$ years $4^{4}$. Not only are we beginning to understand more about metabolism during tumour initiation and metastasis, and its context dependence, but we are also gaining more insights into the metabolism of cells in the TME, including immune cells.

There is a strong argument that prevention is better than cure. Although in many cases, we may not be able to prevent cancer entirely, early detection may improve outcomes. One way this might be achieved is through synthetic biomarkers to amplify disease signals.

Importantly, equity between high-income countries and low- and middle-income countries, as well as equity between populations within high-income countries in terms of cancer prevention, detection and care must be achieved. In addition, we must work harder to make the cancer research community more inclusive.

While we hope that the next 20 years will bring successful strides towards making all cancers manageable, rather than fatal, diseases, there are many obstacles to reaching that point. Perhaps not surprisingly, it seems that, just as we think we have something all figured out, more questions arise. Indeed, the editorial team has often commented that our favourite cancer research papers are those that raise more questions than they answer. Human biology is inherently complex and dynamic, and we are only at the cusp of understanding how these complexities influence tumour development. For example, tissue specificity of oncogenes; dissemination, dormancy and metastatic outgrowth of tumour cells; systemic changes in metabolism and immunity; and the ageing process are all key future areas that are highlighted in articles in our Collection.

Last but not least, we must thank everyone who has played a part in creating Nature Reviews Cancer and developing it into a resource that is trusted by the cancer research community. Thank you to that community for supporting the journal through reading, writing and/or peer reviewing articles, thank you to all of the editors past and present, who have shaped the journal into what it is today through many hours of hard work and dedication, and thank you to those working behind the scenes: art editors, copy editors, production editors and editorial assistants to name but a few.

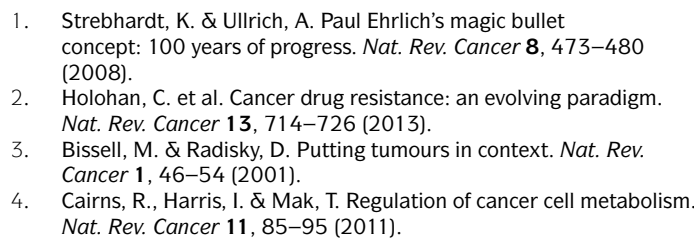

